\title{
Drug Distribution Analysis of Controlling the Ebola Epidemic Based on the Intelligent Systems
}

\author{
Ning Han \\ School of North China Electric Power University, Baoding 071000, China; \\ hannahning@163.com
}

Keywords: Drug distribution, Ebola, Intelligent systems

\begin{abstract}
This paper mainly analyzes how to ease the Ebola epidemic and develops a drug distribution program, including the transportation destinations of drugs and other circumstance. On the basis of possessing the drugs, we need to build a realistic, sensible, and useful method in the prevention and control of Ebola. There are four steps: calculating the drug demand index; making sure the better plan; getting manufacture speed and finding the short supply and oversupply drug distribution scheme. With the research of the Intelligent Systems Analytic, we can clearly develop two program from the analysis of the relationship between supply and demand in the future. In short supply, calculate the drug demand indexes of the three countries by considering the total number of patients and the prevalence trends. When oversupply, arrange distribution program to meet the demand. Taking effective programs which are calculated in the method is better to control the epidemic.
\end{abstract}

\section{Introduction}

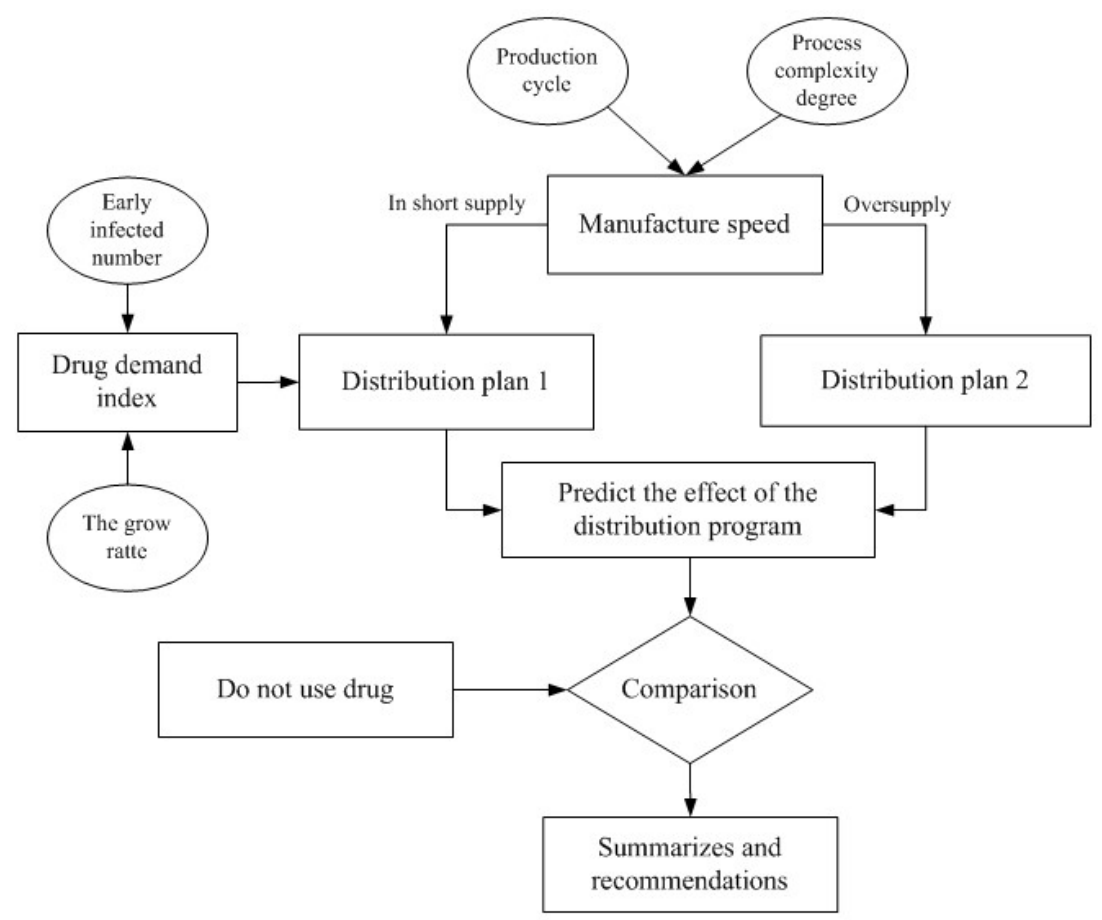

Fig. 1 The flow chart of drug distribution program

\section{Step 1. Calculate the number of patients in the early period.}

By using the drug demand index to calculate the number of infected people who is in the early stage and the recent growth rate.Firstly, the number of infected people who is in the early stage we can predict the infected number prevalence trends over time by analyzing the model one results. Then we can use the following equation to obtain the number of infected people who is in the early stage.

$X_{\mathrm{i}}=f(t)-f(t-1) \mathrm{t}$ is week number.

Next, the recent growth rate we can predict the infected number prevalence trends over time by analyzing the model one results. Calculating the growth rate of the number of patients Recently in the 
countries, the goal is to reflect the urgency of the country to drug treatment .Studies of this parameter is intended to more effectively control the spread of Ebola disease rate, when a region rapidly increased the number of patients should be more drug delivery as soon as possible to prevent the number of patients jumped. The growth rate of the number of patients $\eta^{-}$is equal to the national average the per week growth rate $\eta \mathrm{ij}$ during the recent month. Formulas are as follows:

$$
\begin{gathered}
\eta_{i j}=\frac{f(j)-f(j-1)}{\mathrm{j}-(\mathrm{j}-1)} \\
\bar{\eta}=\frac{\sum_{\mathrm{i}=1}^{4} \eta_{i j}}{4}(j=\mathrm{N}, \mathrm{N}-1, \mathrm{~N}-2, \mathrm{~N}-3)
\end{gathered}
$$

Because of different dimension of the number of infected people who is in the early stage and the recent growth rate, evaluation matrix needs to normalize. We stipulate that the number of infected people who is in the early stage is the main factor, the recent growth is the minor factor. It is realistic based on the actual situation. So we can give two factors empowerment value, the values are 0.7 and 0.3 , the formula is as follows:

\section{$d_{i}=0.7 Y_{i}+0.3 \eta_{i j}$ \\ Step 2. Summarize drug production rules access to information and developing a drug distribution program based on drug production.}

We choose the best vaccine ZMapp.It is now considered the most effective drug to stop Ebola and cure patients whose disease is not advanced. However, affected by the complex production process,the speed of Ebola virus drugs manufacturing is slow. Pharmaceutical raw materials need to be extracted from tobacco and tobacco infects antibody that was transformed. Then the tobacco can produce enough materials after a week. Finally, materials are refined to get enough antibodies. Due to the above describes production process, leading to the account of vaccine is extremely small and the state is currently out of stock.

So we think the speed of production is divided into two parts. The first part is vaccine just into product that production is extremely small because technology is not mature enough. Moreover, the production was restricted by raw materials and so on. So, we assume that the slope of production was considered small linear growth. Another part is over time and the technology continues to mature. And the production of vaccine was increased by exponential growth. Then the production is enough to meet the demand and we can adjust the output according to the demand.

On the other hand, the wider spread of Ebola, more focus made on Ebola. Manufacture speed will increase.

Based on the above data, we think the the manufacture speed is as shown in the below: Fig.2.

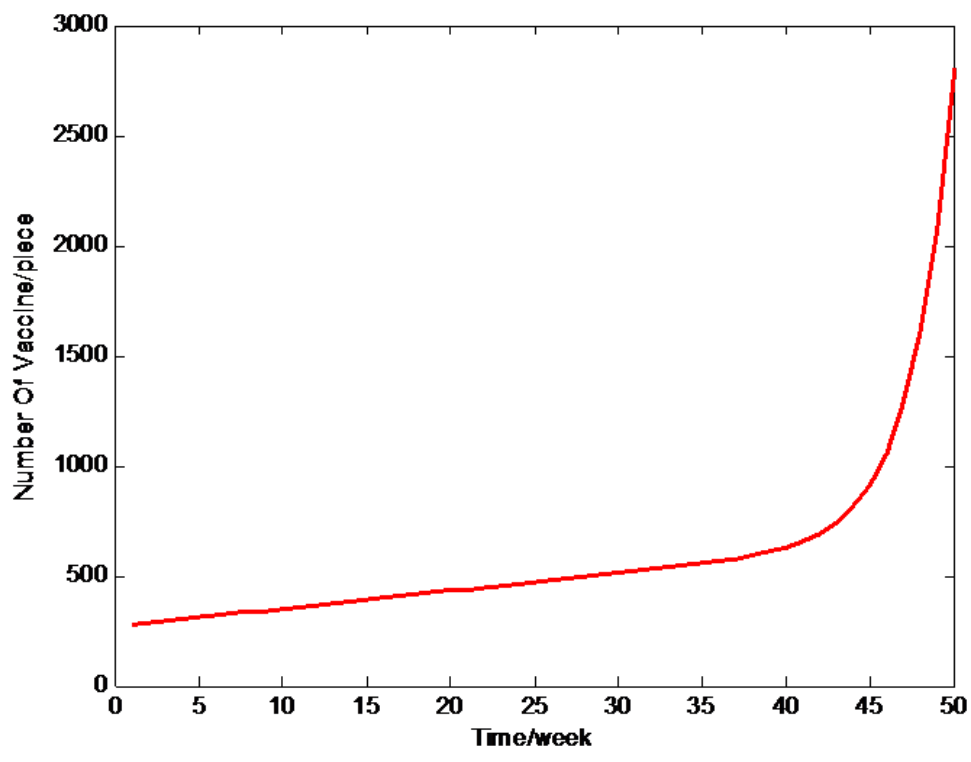

Fig. 2 Manufacture speed 


\section{Step 3. Calculate the effects arising from the drug distribution program.}

We get changes of the supply and demand with the passage of time by the calculation above.Drug distribution scheme is divided into two cases according to the relationship between supply and demand, i.e., the drug in short supply or oversupply.

1) The drug is in short supply

In the case of short supply, Some areas are not be able to get enough drugs. To a reasonable allocation of the limited amount of drugs, we conclude the di, which is influenced by the local early infected number and the spread trend.So when the amount of drug is small, the drag assigned to each country is obtained by the following formula.

$$
\mathrm{Zi}=d i \times M j
$$

2) The drug is oversupply

In the case of oversupply, we can distribution enough drugs according to the demand. Each country gets enough drugs.

\section{Summary}

We use rational drug distribution scheme, effectively slowing the spread of the Ebola epidemic when the drug yield is little. With the increasing amount of drug production, alleviating of the epidemic is growing. Probably in 43th week, the number of patients rapid decline in West Africa. In 48 week, the epidemic has been completely eliminated.The comparison chart as below Fig.3.

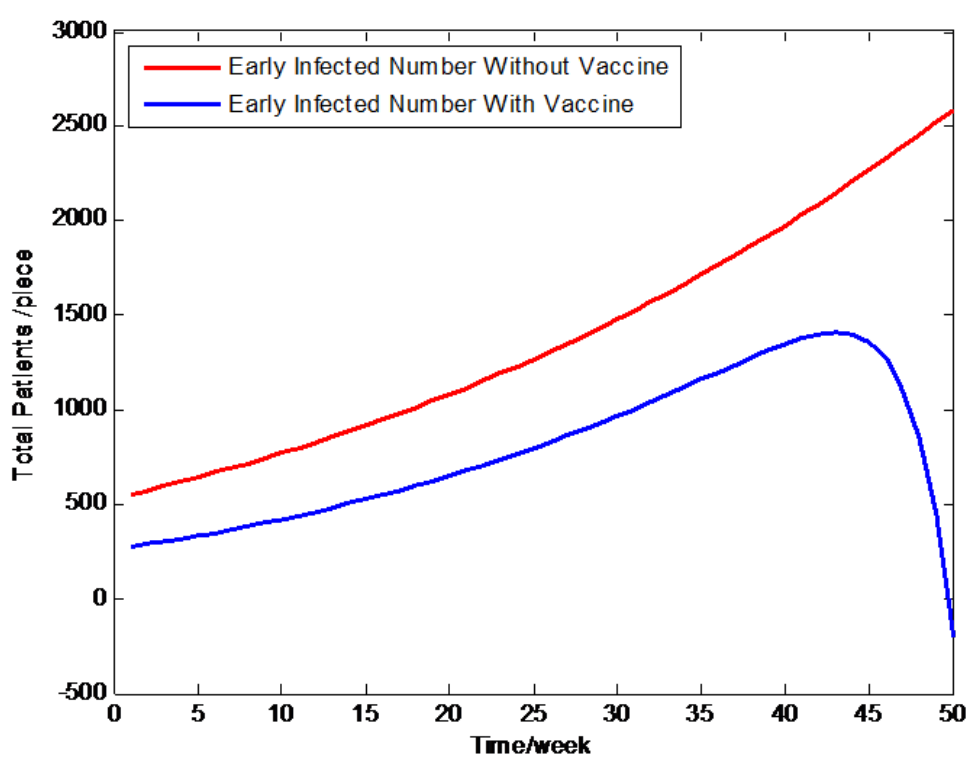

Fig. 3 The comparison chart

\section{References}

[1] Wang Xiaoli, Cao Zhidong, increased military, et al. application seir model to predict the 2009 influenza pandemic hln1 trend [J] International Journal of Virology journal, 2011, 18(6):161-165.

[2] Information on: http://en.wikipedia.org

[3] Information on: http:// wenku.baidu.com 